\title{
LA FINALIDAD DE LA ENCARNACIÓN DE VISNU COMO KRSNA DE ACUERDO CON EL BĀCACARITA DE BHĀSA
}

\author{
LUIS GONZÁLEZ REIMANN
}

ENTRE LAS FUENTES HINDÚES que describen los primeros años y las proezas infantiles de Krsna, el Bălacarita, una obra de teatro escrita por Bhāsa, es considerada generalmente como una de las dos más antiguas, siendo la otra el Harivamsa (ca. siglo VI d.C.), un apéndice al Mahābhārata escrito en el estilo de los purānas. Los otros textos importantes sobre el tema son el Visnu Purāna (ca. siglo V d.C.) y el Bhāgavata Purāna (ca. siglo IX d.C.).

La época de la vida de Bhāsa, como muchas otras fechas en la historia india, es una cuestión controvertida. Pusalker, en su estudio sobre Bhāsa, argumenta en favor de una datación más temprana que el periodo del imperio Maurya y la ubica alrededor del siglo V o IV a.C. ${ }^{1}$ No obstante, ya en el prólogo al libro de Pusalker, A.B. Keith discrepa con una estimación tan temprana. ${ }^{2}$ Woolner, que tradujo las obras de Bhāsa, parece aceptar como posibles fechas los siglos segundo y tercero d.C. o el séptimo e incluso después. ${ }^{3}$ Es interesante que en el Mahābhāsya, una obra escrita por el gramático Patañjali (ca. siglo Il a.C.), se mencione una representación escénica de la muerte de Kamsa por Krsna, ${ }^{4}$ evento que, por supuesto, es el tema central de la historia. Sin entrar en esta discusión, consideraremos, para los presentes propósitos, al siglo $\mathrm{V} \mathrm{d}$.C. como una fecha general razonable para Bhāsa. ${ }^{5}$

Sin embargo, la datación de éste no es el único problema, ya que la propia autoria de las obras ha sido cuestionada. Fue el estu-

${ }^{1}$ Pusalker, Bhāsa - a study, p. 89.

2 Ibid., p. vii.

3 Woolner, Thirteen Plays of Bhāsa, p. vi.

4 Patañjali sobre Pānini 3.1.26; de acuerdo con Preciado, The Krsna Cycle in the Purānas, p. 30. pitulo 3.

${ }_{5}$ Para una visión más completa de las diferentes opiniones véase Pusalker, Bhāsa, ca- 
dioso moderno Ganapati Sāstri quien atribuyó a Bhāsa las 13 obras que se le adjudican, entre ellas el Bālacarita, ${ }^{6}$ y Pusalker está de acuerdo en que todas fueron escritas por la misma mano. ${ }^{7} \mathrm{De}$ vádhar, quien editó el texto sánscrito, es de diferente opinión. De acuerdo con él, las obras no son la producción de un solo escritor y no pueden ser consideradas como obra de Bhāsa. ${ }^{8}$ También se ha sugerido que, incluso si fueron escritas por el mismo autor, los originales pudieron haber sido corregidos en fecha posterior. ${ }^{9}$ Considerando que la autoria real de la obra no es directamente relevante para nuestros fines, y que los fragmentos del Bälacarita que podrian ser considerados como adiciones ${ }^{10}$ no son cruciales para nuestra exposición, aceptaremos a Bhāsa como autor del Bālacari. t $a$, por razones de simplicidad.

Antes de seguir adelante debemos señalar que en la obra a Krsna se le llama por ese nombre sólo en dos ocasiones, y en una de ellas en forma indirecta. ${ }^{11} \mathrm{~A}$ partir del interludio del tercer acto, donde se explica el origen del nombre, se le llama Dāmodara. ${ }^{12}$ En la literatura temprana, este nombre sólo se menciona con referencia a Krsna en uno de los Dharma Sūtras escritos probablemente en el siglo IV a.C.13

En el Bālacarita, el propósito de la encarnación de Krsna se afirma con claridad desde el comienzo. En el acto I, el sabio celestial Nārąa explica que ha venido a la tierra para ver al señor Nārāyana, quien habia nacido como Krsna en el linaje de los Vrsni, "con el

6 Devadhar, Bhāsanātkacakram, p. 5.

${ }^{7}$ Pusalker, Bhāsa, pp. 22-23.

${ }^{8}$ Devadhar, p. $x$.

${ }^{9}$ Woolner parece favorecer esta opinión; Thirteen Plays, pp. vii-viii.

10 Véase Woolner, vol. 2, pp. 109.111.

11 Sankarsana se refiere a él como Krsna en 4.3. Cuando Cakra, personificación del disco de Visnu, se presenta dice que es el disco de Krsna (1.22). Los otros nombres usados para referirse a Krsna en la obra son: Visnu (cinco veces), Nārāyana (tres veces), Vāsudeva (dos veces) y Hari, Purusottama y Madhusūdana (una vez cada una).

12 Dāmodara significa "el que tiene una cuerda (dāman) alrededor de su vientre (udara) ${ }^{n}$. El nombre se deriva de una de sus proezas de infancia (a la edad de un mes, de acuerdo con Bhāsa). Su madre lo ató a un mortero con una cuerda porque había estado robando comida de muchas casas, pero el niño taló dos árboles con ella (demonios disfrazados en la versión de Bhāsa), y fue llamado Dāmodara a partir de entonces por las pastoras de su aldea.

${ }^{13}$ Baudhāyana Dharma Sūtra 2.5.9.10; de acuerdo con Preciado (The Krsna Cycle, p. 20), quien refiere a A. A. Macdonell como su fuente para esta fecha. Para un cuadro que presente las menciones tempranas de los diferentes nombres asociados con Krsna, véase Precia. do, p. 38. 
propósito de matar a Kamsa para beneficio de todo el mundo". ${ }^{14}$ Kamsa, que es tío de Krsna, ha usurpado el trono de Mathurā a su propio padre, el rey Ugrasena, a quien puso en prisión. Debido a una maldición, Kamsa sabía que un hijo de Vasudeva y su mujer Devaki estaba destinado a matarlo y, por lo tanto, habia forzado a aquél a que aceptara entregarle a todos sus hijos, para eliminarlos tan pronto como nacieran. Cuando Krsna nació soplaron fuertes vientos, un terremoto sacudió la tierra y apareció un cometa, signos todos a los que Kamsa consideró ominosos. Su astrólogo y el sacerdote de su corte le dijeron que estos portentos significaban que "el ser eterno que vive en el cielo ha venido al mundo de los hombres por alguna razón" 15 y Kamsa, con una sensación de fatalidad inminente se muestra ansioso de inmediato y quiere descubrir el propósito de tal nacimiento (janmaprayojanam). ${ }^{16} \mathrm{~A}$ pesar de los esfuerzos de Kamsa, Krsna lo mata y poco antes de hacerlo declara que su nacimiento entre los mortales podía resultar inútil si no cumplía su tarea:

\footnotetext{
Mi nacimiento entre los mortales seria inútil y mis proezas en el campamento de los pastores y hoy en la ciudad no me harán feliz hasta tanto no derribe en combate y arrastre tras de $\mathrm{mi}$ al malvado Kamsa, que en ot ro nacimiento fue un demonio (asura). 17
}

Una vez que Krsna hubo matado al rey, Vasudeva proclama al pueblo de Mathurā que fue el propio dios Visnu quien llegó hasta ellos con el propósito de matar a Kamsa, ${ }^{18}$ y se refiere a él como el destructor de Kamsa. ${ }^{19}$

Según se afirma en el verso recién citado, la muerte de Kamsa forma parte de un plan más amplio, ya que éste habia sido un de-

14 'Lokahitārthe kamsavadhārtam' (1.6); esta afirmación es repetida por Vasudeva, el padre de Krsna, en 1.19. (2.10).

15 'Bhūtam nabhastalanivāsi ... nityam kārtāntarena naralokam iha prapannam'

162.11.

17 'Martyesu janma viphalam mama tāni ghose karmāni ca adya nagare dhrtaye na tāvat'/ yāvan na kamsahatajam yudhdhi pātayitvã janmāntarāsuram parikarsayami ' $/ /(5.6)$.

18 'Kamsārtham svayam iha visnur ājagāma' (5.13).

19 'Kamsāntaka' (5.14). 
monio en un nacimiento anterior ${ }^{20}$ y Bhāsa describe a'Nárāyana/Nisnu como "el destructor de demonios", 21 "el destructor de la fuerza de los demonios" 22 " "el que mata hordas de demonios y descendió para la protección de la gente". ${ }^{23}$ Hay al respecto un verso interesante en el acto III del Bălacarita. Cuando el toro Arista (que es un demonio disfrazado) está a punto de emprender batalla contra el niño Krsna, lo reconoce como Visnu y se lamenta asi:

Dondequiera que nazcamos, allí también [nace]

el sostenedor de los tres mundos (Visnu).

Madhusūdana (Visnu) está [alli] a fin de matar

a los demonios. ${ }^{24}$

Así, si una de las funciones de Visnu es destruir demonios y él aparece con ese propósito dondequiera que se muestre, entonces la muerte de Kamsa a manos de Krsna es parte de esa función. Nótese, sin embargo, que la obra nunca afirma que la muerte de los demonios en general sea el propósito del nacimiento de Krsna (este verso sería precisamente el que más se acerca a esa afirmación), sino que es muy especifica en su afirmación de que es la muerte de Kam. sa lo que Krsna debe realizar. También es interesante que Kamsa no sea mencionado como un demonio, sino como alguien que ha sido un demonio en un nacimiento anterior.

Sin embargo, el niño Krsna mata muchos otros demonios en la obra. Al pasar en el Interludio al acto III, se presenta una lista de ellos. Son los siguientes:

1. La demonio Pūtaná, quien había adoptado la forma de la madre adoptiva de Krsna y habia intentado amamantarlo con veneno. ${ }^{25}$

2. El demonio Sakata, que se había convertido en un carro. boles. ${ }^{26}$

3. Yamala y Arjuna, que habian adoptado la forma de dos ár-

20 El término "demonio" será utilizado para traducir tanto 'asura' como 'dānava' y 'amarāñ'.

21 'Daityānām vidhanakarah' (1.6).

22 'Asuraviryahantä (1.7).

23 'Raksanārtham prajānām asurasamitihantā visnur adya avatïrnah' (1.9).

24 Yatra yatra vayam jārās tatra tatra trilokadhrt / dānavānām vadhārthāya vartate madhusūdanah $/ /(3.13)$.

25 Los padres adoptivos de Krsna eran el pastor Nandagopa y su esposa Yasodā.

${ }^{26}$ Cabe mencionar que en ot ras versiones el carro y los árboles no se consideran como 
4. Dhenuka, que llegó disfrazado de burro.

5. Kesin, que se presentó como un caballo.

Curiosamente, la lista incluye tambien la muerte de Pralamba (que habia adoptado la forma de Nandagopa), aunque se menciona que no lo mató Krsna mismo sino Sankarsana (Balarāma), su hermano mayor.

A esta lista debemos agregar: 1) la muerte del toro Arista, descrita en detalle en el acto III; 2) el sometimiento (no muerte) de la gran serpiente Kāliya, que es el tema principal del acto IV; y, finalmente, 3) la muerte de Kamsa, descrita brevemente en el quinto y último acto del Bālacarita.

Los episodios de Arista y Kāliya reciben gran importancia y son descritos en detalle; haremos algunos comentarios sobre ellos. Son similares en varios aspectos, e incluso Bhāsa se refiere a ellos en forma paralela, pero también presentan importantes diferencias.

La arrogancia parece ser una de las características más importantes de la personalidad de los demonios, si no la más significati. va, y Arista y Kāliya no son la excepción. Ambos se jactan de su fuerza y declaran que matarán fácilmente al niño. Krsna, antes de enfrentarse a cada uno de ellos asegura de forma rotunda y con las mismas palabras en ambas ocasiones, que "acabará con su orgu1lo." 27 Cuando Arista trata de intimidarlo Krsna se para en una pier na y desafía al toro a que lo derribe. De forma similar, cuando Kāliya amenaza con abrasar a Krsna con sus poderosas llamas, éste lo desafía a que queme uno solo de sus brazos. Ambos fallan en su intento y Krsna se burla de ellos con estas palabras: "Ésà es la fuerza de que estáis orgullosos." ${ }^{28}$ Ambos reconocen entonces que el niño es Visnu, pero el resultado es diferente en cada caso. Arista decide combatir de todos modos, porque sabe que si Visnu lo mata él irá al cielo, ${ }^{29} \mathrm{y}$ de este modo Krsna lo mata. Kāliya, por su parte,

demonios y que los incidentes se presentan simplemente como prodigios de fuerza. Para referencias y una discusión sobre la importancia de estos dos enfoques, véase Preciado, The Krsna Cycle, pp. 67 y ss.

27 'A ham asya darpaprasamanam karomi'; 3.4 (para Arista), 3.16 (para Kāliya).

28 'Anena vīryena bhavān garvitah'; 3.12 (para Arista), 4.11 (para Kāliya).

293.14. 
ruega a Krsna que lo perdone, diciendo que su transgresión fue causada por la ignorancia, y tras ser humillado y conminado a no causar problemas a nadie, consigue el perdón.

Algo interesante respecto de Arista es que cuando encuentra a Krsna dice que "ha tomado la forma de un toro con el propósito de matar a su enemigo", ${ }^{30}$ quien claramente es Krsna. En lo que parece una curiosa inversión de papeles, el demonio Arista es retratado aquí, como habiendo encarnado, en cierto sentido, con el propósi. to de matar a Visnu en la forma del niño Krsna. También resulta sorprendente que inmediatamente después de matar a Arista, Krsna se refiera a él como el señor de los demonios ('dānavendra'), ${ }^{31}$ en contraste con el epíteto de Krsna, "maestro de los dioses," que había sido utilizado antes por Nārada. ${ }^{32}$ Esto podría, tal vez, ser considerado simplemente como una forma por medio de la cual Bhāsa intenta hacer más precisa la escena ya que se trata, después de todo, de uno de los episodios presentados con mayor detalle. Sin embargo, en otras versiones de la historia, quien es el líder de los demonios es Kamsa, lo cual es consistente con que su muerte sea el propósito del descenso de Visnu a la tierra. En la versión del Visnu Purāna, por ejemplo, Visnu encarna como Krsna para librar a la tierra de los incontables demonios que la cargan con su peso, y Kamsa es mencionado como su jefe. ${ }^{33}$ En el Bălacarita no hay indicación de que Kamsa sea líder de otros demonios.

Hay también algo más que merece advertirse sobre la versión de Bhāsa del episodio de Arista. Ya hemos citado el verso en el que el toro, tras comprender quién es el niño, dice que Visnu nace dondequiera que nacen los demonios a fin de matarlos. Este verso muestra una interesante semejanza con los dos bien conocidos versos de la Bhāgavad Gitā en que Krsna le explica a Arjuna su finalidad al nacer en este mundo. Dice Krsna:

Cuando dharma decrece, Bhārata, y adharma
se incrementa, me creo a mi mismo. Nazco de
yuga en yuga para la protección de los buenos

30 'Satror vadhărtam upagamya vrsasya rūpam' (3.5).

313.15.

32 'Guruh surănām' (1.6).

${ }^{33}$.VP 5.1, y véase también el comienzo de 5.4 , donde Pralamba, Kesin, Dhenuka, P $\overline{\mathrm{u}}$ tanāy Arista reciben óndenes de Kamsa. Traducido en Wilson, The Vishru Purăna, pp. 396, 404. 
y la destrucción de los malvados a fin de establecer a dharma. ${ }^{34}$

Según se afirma aquí, y ésta se convertiría después en la explicación corriente, la finalidad de la encarnación de Krsna es notoriamente más amplia y tiene una connotación diferente. Luego volveremos sobre esto.

Puede decirse todavia algo más con relación al episodio de Arista. Cuando Arista trata de atemorizar a Krsna, el dios responde afirmando que él no conoce el miedo, y llega a declarar que nació sobre la superficie de la tierra "a fin librar del temor a quienes están afligidos". 35

En cuanto a la serpiente Kāliya, debe señalarse que esta proeza de Krsna es diferente de los otros episodios de matanza de demonios en dos formas, y que esto es aplicable no sólo al Bălacarita sino también a todas las otras versiones del incidente. En primer lugar, nunca se dice que Kāliya sea un demonio; Kāliya es una serpiente. ${ }^{36}$ Podríamos tratar de explicar esto recurriendo al pasaje del Mahă. bhārata que describe cómo los dioses descendieron a la tierra a pedido de ésta (del mismo modo que en el pasaje del Visnu Purāna antes mencionado) para destruir a los demonios que le pesaban demasiado. En la épica se dice que los demonios toman la forma de toda clase de seres ${ }^{37}$ y que cuando los dioses encarnaban "mataban muchos dānavas, rāksasas, gandharvas (sic), serpientes (pannagas) y otros seres devoradores de hombres". ${ }^{38}$ Esto, sin embargo, no explica por qué -si tal era el caso- Kāliya nunca es identificado como un demonio.

La segunda cosa notable acerca de Kāliya es que Krsna no lo mata, a pesar de que aquél sí intenta hacerlo. Antes de la pelea, Krsna proclama sus intenciones con estas palabras: "voy a someter a esta serpiente para beneficio de toda la gente." ${ }^{39}$ Estaría más allá del alcance de este articulo intentar explicar los rasgos que hacen diferente al episodio de Kāliya, pero lo que es interesante para nues-

\footnotetext{
34 'Yadā yadā hi dha rmasya glănir bhavati bhārata / abhyutthănam adharmasya tadāat. mānam srjāmi aham // parirrānāya sādhūnām vināsaya ca duskrtām / dharmasamsthāpanārthāya sambhavāmi yuge yuge' $/ /$, BhG 4.7.8.

35 'Bhitânām abhayam dātum samutpanno mahitale' (3.8).

36 'Uraga' (4.2), 'naga' (4.4).

37 Mbh 1.58. 2532.

${ }^{38}$ Mbh 1.59.5.

39 'Sarvaprajāh itărrtham nāgam me vasam karonu' (4.4).
} 
tros propósitos acerca de la versión de Bhāsa es que entre quienes se benefician de la derrota de Käliya se menciona especialmente a los brahmanes, la casta sacerdotal. Una vez que Krsna hubo derrotado a la serpiente, Sankarsana declara que "habia sido hecho para beneficio de las vacas y de los brähmanas." 40 Esto parece indicar que Bhāsa era parcial en favor de éstos ${ }^{41} \mathrm{e}$ intentaba introducir esto delicadamente en la trama de la obra, al explicar el propósito de la proeza de Krsna.

A partir de lo que hemos visto, podemos decir que el tema de la finalidad de la encarnación de Visnu como Krsna tiene tres capas. Existen tres diferentes propósitos para la encarnación, que pueden ser $-\mathrm{y}$ de hecho lo están- combinados en diferentes formas, y que son los siguientes:

1. Matar a Kamsa, un rey usurpador.

2. Matar a los demonios encarnados que agobiaban a la tierra con su peso.

3. Restaurar el dharma sobre la tierra cuando éste ha decrecido.

En cada caso subyace el tema de la salvaguarda del mundo, algo que concuerda bien con el papel benéfico y protector de Visnu. No obstante, como puede verse fácilmente, cada una de estas metas es más general que la anterior. En el primer caso, se trata de un evento singular y localizado: la muerte de un rey corrupto. En el segundo, quienes deben ser destruidos son todos los demonios. Ésta todavía es una tarea bien definida. El tercer caso, sin embargo, es lo bastante amplio como para abarcar no sólo a los dos primeros sino también a cualquier otro hecho que pudiera ser estimado necesario para el adecuado funcionamiento del mundo.

Los dos primeros aparecen combinados, aunque de forma diferente, tanto en el Bālacarita como en el Visnu y el Bhāgavata Puranas, y el tercero es expuesto en la Gitä y es retomado a partir de entonces en muchos textos. ${ }^{42}$ Comentaremos ahora más detenidamente los tres propósitos antes mencionados de la encarnación de Krsna.

40 'Gobrāhmanahitam Krtam' (4.13).

${ }^{41}$ Esto ya habia sido mencionado por Pusalker; Bhāsa, pp. 18, 355.

42 Esto no significa necesariamente afirmar que la Gitā sea, desde un punto de vista histórico, el primer texto en mencionar esta idea (aunque bien podria ser el caso), sino que ha sido, seguramente, el más influyente. 


\section{La muerte de Kamsa}

El Bālacarita concluye con la muerte de Kamsa y la restauración de Ugrasena en el trono y la historia parece completa, sin necesidad de ninguna proeza posterior por parte de Krsna. Lo que Krsna hace luego es secundario, porque su propósito al descender a la tierra ya ha sido realizado. Sin embargo, en el Mahābhārata, que se ocupa de su vida adulta y sólo se refiere brevemente a sus años tempranos, Krsna dice que habia matado a Kamsa por deber a su familia, ${ }^{43}$ porque Kamsa estaba persiguiendo a sus parientes (bhāndavān)..$^{44}$ En el quinto libro dice que lo hizo porque queria beneficiar a sus familiares. ${ }^{45}$ En estos pasajes de la épica no se menciona a Kamsa como un demonio y, lo que es más importante, todo el asunto suena como solo uno de los muchos episodios de la infancia de Krsna, casi una cuestión familiar a la que no se da particular importancia. ${ }^{46}$

\section{La destrucción de los demonios que agobian la tierra}

Éste es un tema muy importante en la mitologia de la épica y de los purānas. El antiguo conflicto védico entre los dioses y los demonios tiene ahora como escenario la tierra, donde ambos bandos han encarnado. El Mahābhārata explica cómo los demonios encarnaron como toda clase de seres (camellos, vacas, caballos, etc.) en un intento de convertirse en dioses. ${ }^{47}$ Algunos de ellos eran reyes, todos eran fuertes y arrogantes, $y$ "oprimian con energia a los brahmanes, los ksatriyas, los vaisyas, e incluso a los sudras y otros seres". ${ }^{48}$ La tierra pidió entonces ayuda al dios Brahma, porque los

\footnotetext{
43 'Jñātikāryam mayā krtam', Mbh 2.13.34. En 2.55.6.7 se afirma que Krsna mató a Kamsa a pedido de los andhakas, los yādavas y los bhojas y que cuando lo hizo sus familiares (jñ̄ätayah) fueron felices durante cien años.

44 Mbh 2.13.29.

45 'Jñ̄âtinām hitakāma', Mbh 5.126.37. En estas citas, los términos 'jn̄̄ati' y 'bhāndava' pod rian ser tomados no simplemente como parientes of familiares, sino "su propia gente" en un sentido más amplio.

${ }^{46}$ En el Mbh. 5.128.45-49, se mencionan muchas de las hazañas de Krsna, entre ellas la muerte de Arista y Dhenuka (sin que se mencione que fueran demonios), y la muerte de Kamsa asi como la de otros reyes.

47 Todos los pasajes de la épica que serán mencionados o a los que se hará referencia están contenidos en Mbh 1.58.25-1.59.6.

${ }^{48} \mathrm{Mbh}$ 1.58.32.
} 
demonios eran demasiado pesados para ella y él cumplió ordenando a los dioses que nacieran para "quitar el peso de la tierra". ${ }^{49}$ Entonces, los dioses (incluyendo a Visnu):

\footnotetext{
...descendieron del cielo a la tierra en el orden debido para destruir a los demonios y para beneficio de todo el mundo.

...nacieron en estirpes de brāhmanas y en familias de reyes y rsis de acuerdo a su deseo.

Mataron a muchos dānavas, rāksasas, gandharvas, serpientes (pannagas), y otros seres devoradores de hombres.

Los danavas, raksasas, gandharvas y serpientes no los mataron porque eran fuertes incluso en su infancia. 50
}

Como sabemos, la muerte de los demonios desempeña una función importante en el Bālacarita, pero es interesante advertir que la obra no menciona la necesidad de aliviar el peso de la tierra. El único lugar donde se puede encontrar una posible referencia a esto es cerca del final, cuando Narada declara, después de que Krsna mata a Kamsa, que "la tierra ha sido protegida con la destrucción de este demonio". ${ }^{51}$ En el Harivamsa este tema está presente y el texto se refiere explicitamente a la necesidad de Visnu de librarse de los reyes que estaban haciendo que la tierra se hundiese como un bote. ${ }^{52}$

Algo más que deberia ser mencionado es el hecho de que en la versión de la épica, no se nombra jamás a Kamsa, mientras que cuando se cuenta la misma historia en el Visnu Purāna, se lo describe como el lider de los demonios. Esto convierte su muerte en la meta principal de la encarnación de los dioses, encabezados por Visnu, ${ }^{53}$ lo cual podría indicar que la historia de la muerte del perverso rey Kamsa a manos de su sobrino Krsna tiene un origen diferente, y que aquí estaría siendo incorporado dentro del amplio plan de la destrucción de los demonios por parte de los dioses, para aliviar la carga de la tierra.

Bhandarkar diferencia claramente entre las dos metas y hace uso de esto en apoyo de la teoría que sostiene que el niño Krsna, que

49 'Bhūmer nirasitum bhāram', Mbh 1.58.46.

${ }^{50} \mathrm{Mbh}$ 1.59.3.6. El tercero de estos versos ya habia sido citado.

51 'Anena asuranăsena mahi pariraksitâ' (5.18).

${ }^{52}$ HV. 41.17-18.

53 Véase la referencia en Wilson, The Visnu Purana, ya mencionado. Para otras referencias, véase O'Flaherty, The Origins of Evil in Hindu Mythology, p. 260. 
encontramos en los textos que hemos mencionado, es el resultado de la unión de dos figuras diferentes: Krsna Vasudeva, el hijo de Vasudeva y matador de Kamsa; y Krsna Gopāla, el dios pastor que siendo niño mató numerosos demonios y realizó otras proezas. ${ }^{54}$ Bhandarkar cita como evidencia el hecho de que en la sección $\mathrm{Na-}$ rāyaniya del Mahābhārata, la encarnación como Vasudeva "se menciona como asumida para la destrucción de Kamsa, pero no de ninguno de los demonios a los que el pastor (gopāla) Krsna mató en el campamento de los pastores". 55

Antes de pasar a discutir la tercera finalidad de la encarnación de Krsna haremos algunas observaciones adicionales sobre el tema del peso que sentía la tierra debido a la presencia de tantos demonios. Aunque el tema no sea retomado en el Bălacarita, la obra de Bhāsa le da cierta importancia a lo que podría considerarse como la contraparte de este tema: el gran peso del niño Krsna.

Cuando Vasudeva recibe al niño de su esposa Devakí para llevarlo a un lugar seguro, fuera del alcance de Kamsa, inmediatamente se sorprende de lo pesado que es el niño, y alaba a Devakĩ por haber sido capaz de soportarlo. ${ }^{56}$ Poco después, tras cruzar el río Yamuna, convence a su amigo, el pastor Nandagopa, de que se lleve al niño y lo críe como su propio hijo. Nandagopa le entrega entonces a su hija, muerta al nacer, para que pueda engañar a Kamsa con ella. Cuando Nandagopa trata de sostener al niño, le fallan los brazos y es sólo cuando el niño se hace más liviano, por intervención divina, que puede finalmente cargarlo. En ambos casos se dice que el niño es pesado como una montaña. ${ }^{57}$ Es interesante que tan pronto como Vasudeva emprende el regreso a la ciudad de Mathurā, la niña vuelve a la vida y también adquiere un enorme peso. Más tarde, cuando Kamsa trata de matarla, se convierte en la diosa Kār. tyāyani (Durgā) y predice su destrucción. ${ }^{58}$

\footnotetext{
54 Bhandarkar, Vaisnavism, Saivism and Minor Religious Systems, p. 35.

${ }^{55}$ Bhandarkar, ibid. El problema de si la historia de Krsna es o no una combinación de estas dos figuras sigue abierto; véase Hawley, "Krsna", passim.

561.12.

57 Vasudeva dice: "este niño es pesado como la cadena de los Vindhya y el monte Mandara" (vindhyamandarasaro ayam balah), 1.12; y Nandagopa: "el niño es como el monte Mandara" (mandarasadrsam balakam) 1.21 .

$58 \mathrm{La}$ inclusión de la diosa Durgā en la historia del nacimiento de Krsna parece innecesa ria y un poco artificial, y el tono del pasaje del Harivamsa que trata acerca de su participación (véase HV; pp. 47-54, y ss.; el "Aryastava") indica que se trata de un medio de incor-
} 
En el Bhāgavata Purāna hay dos incidentes en los que se dice que el niño Krsna es muy pesado y se lo compara otra vez con una montaña. ${ }^{59} \mathrm{Y}$ en el Harivamsa, cuando se menciona que las montañas ("las sostenedoras de la tierra") temblaron en el momento del nacimiento de Krsna, el comentarista Nílakantha explica que esto sucedió "debido al peso del Señor" ${ }^{60}$

Es interesante observar cómo la noción de peso que, por extensión conlleva las ideas de importancia, respetabilidad y grandeza, es usada aquí en un sentido literal.

\section{La restauración de dharma}

Mientras las dos primeras metas están incluidas en la obra de Bh̄sa, este tercer tema está ausente del Bālacarita, donde el término dharma ni siquiera se usa.

Hemos citado los celebrados versos de la Bhāgavad Gitā que afirman que Krsna encarna yuga tras yuga para restaurar el dharma cuando éste declina. En la Gitāa, la encarnación del dios es investida con una mayor significación cósmica y está relacionada con los ciclos de incremento y disminución de dharma: los yugas. Esta idea po. ne el propósito de la encarnación dentro de un contexto más amplio y de más largo alcance. Puede así, como lo hemos mencionado, incorporar casi cualquier cosa, especialmente la muerte de los demonios, ya que ellos pueden ser convertidos muy fácilmente en responsables de la pérdida de dharma. Esto es precisamente lo que el Mārkandeya $\mathrm{Pu}$. rāna hace cuando explica cómo Hari (Visnu), como una de las formas de Nārāyana, aparece sobre la tierra cada vez que dharma declina y adharma se incrementa. En la versión del Mārkandeya, se afirma con claridad que son los demonios quienes obstruyen a dharma. ${ }^{61}$

\footnotetext{
porar su culto en el rebaño de los fieles de Visnu-Narayana. Sin embargo, su función mitológica como matador de demonios se acomoda bien dentro de la historia.

${ }^{59} \mathrm{BhP} 10.7 .18-19$ y 10.7.20, 27.28, según cita Preciado, The Krsna Cycle, pp. 71, 76

60 'Bhagavaro bhārena', sobre HV 2.4.15, edición de Bombay (48.14 en la edición critica). "Las sostenedoras de la tierra ('dharanidharāh')" son probablemente las montañas, aunque Nilakantha prefiere leer este compuesto como una referencia a la serpiente Sesa y otros seres míricos.

${ }^{61}$ MP 4.44.58, de acuerdo con Dimmitt y van Buitenen, quienes traducen el pasaje en su Classical Hindu Mythology, p. 66.
} 
Pero incluso si la restauración de dharma puede dar cabida a diferentes metas, hay todavia una gran distancia entre decir que un dios como Krsna desciende a la tierra para un evento específico y limitado (la muerte de Kamsa), y declarar que aparece en la tierra cada vez que dharma pierde fuerza. Ya no se trata aqui de desempeñar una determinada tarea, sino de supervisar el adecuado funcionamiento de todo el orden social e intervenir cada vez que resulte necesario. A su tiempo, con el crecimiento del movimiento devocional bhakti, el énfasis se traslada a una relación más personal entre el dios y el devoto, y el papel de Krsna se convierte en cuidar y proteger a quienes lo veneran. En este punto, la muerte de Kamsa y de todos los demonios no sólo se hace inconsecuente, un mero episo. dio épico, sino que puede incluso ser completamente olvidado.

Hemos visto cómo el propósito de la encarnación de Krsna ha sido explicado en $t$ res formas diferentes y cómo estas tres explicaciones han sido combinadas o enfatizadas de diferentes formas. Es dificil establecer si estuvieron alguna vez completamente separadas o en qué punto y bajo cuáles circunstancias fueron fundidas. El estudio del desarrollo de la figura de Krsna tiene muchas facetas y éste es un aspecto de él que puede echar alguna luz sobre la cuestión. Para esto el Bālacarita sigue siendo una muy valiosa fuente temprana.

\section{BIBLIOGRAFIA}

Bhandarkar, R.G. Vaisnavism, Saivism and Minor Religious Systems. Varanasi: Indological Book House, 1965 (1913).

Buitenen, J.A.B. van (trad.) The Mahābhārata, vols. 1-3. Chicago: University of Chicago Press, 1973-1978.

The Bhāgavad Gitā in the Mahäbhārata. (Texto y traducción.) Chicago: The University of Chicago Press, 1981.

DevadhaR, C.R. (comp.) Bhāsanātakacakram. Plays Ascribed to Bhāsa, Ori ginal Thirteen Texts in Devanāgari. Poona Oriental Series, núm. 54. Poona: Oriental Book Agency, 1962.

Dimmit, Cornelia y J.A.B. van Buitenen. Classical Hindu Mythology, a Reader in the Sanskrit Puranas. Filadelfia: Temple University Press, 1978.

Harivamsa (edición crítica), vol. 1, Parashuram Laksman Vaidya, editor general. Poona: Bhandarkar Oriental Research Institute, 1969.

Harivamsa (edición de Bombay). Shriman-Mahäbhāratam, parte ViII, Harivamshaparvan. Ramachandrashāstri Kinjawadekar (comp.), texto 
sánscrito con el comentario de Nilakantha. Impreso y publicado por Shankar Narhay Joshi. Poona: Chit rashala Press, 1936.

HawleY, John Stratton. "Krsna", en The Encyclopedia of Religion, vol. 8, pp. 384-387. Mircea Eliade, editor jefe. Nueva York: Macmillan, 1987.

Mahābhārata (edición crítica), vols. 1, 2 y 6. Editado por Vishnu S.Suthankar (vol. 2, junto con S.K. Belvalkar). Poona: Bhandarkar Oriental Research Institute, 1933-1944.

O'FlaherTY, Wendy Doniger. The Origins of Evil in Hindu Mythology. Berkeley: University of California Press, 1976.

Preclado Solis, Benjamin. The Krsna Cycle in the Purānas, Themes and Motifs in a Heroic Saga. Delhi: Motilal Banarsidass, 1984.

PusalKer, A.D. Bhāsa - a Study. 2a edición. Delhi: Munshiram Manoharlal, 1968.

Studies in the Epics and Purānas. Bombay: Bharatiya Vidya Bhavan, 1963.

ROCHer, Ludo. The Purānas. A History of Indian Literature, vol. II, fasc. 3. Wiesbaden: Otto Harrassowitz, 1986.

Wilson, H.H. (trad.). The Vishnu Purāna, a System of Hindu Mythology and Tradition, 3a edición. Calcutta: Punthi Pustak, 1972.

WoOlNer, A.C. y Lakshman Sarup (trad.). Thirteen Plays of Bhāsa. Panjab University Oriental Publications, núm. 13, 1930. Reimpreso (dos volúmenes en uno). Delhi: Motilal Banarsidass, 1985. 